\title{
Grammatical and Associative Constraints in Sentence Retention 1
}

\author{
KELYN H. ROBERTS \\ University of Michigan, Ann Arbor, Michigan 48104
}

\begin{abstract}
The frce learning technique was used to test several hypotheses about sentence processing in recall. Sentence complexity (mean depth), syntactic type (active, passive), and subjectobject association direction were found to be significant factors in over-all sentence recall. Recall error patterns showed differences between syntactic type and between association conditions. In addition, Ss showed evidence for an incomplete transformational process.
\end{abstract}

The present experiment was conducted against a background of controversy about both the psychological structure and the mode of processing sentences. The aim of these studies was not so much a resolution of the controversies as an exploration of relevant variables and effects that require explication before a resolution can be proposed. One variable frequently emphasized in previous research has been syntactic type. Mehler (1963) has shown that when length and sentence complexity are uncontrolled, passive sentences are more poorly recalled than actives, with a tendency for passives to be recalled in their active form. The explanation of these phenomena has been couched in terms of transformational rules and $S$ 's memory capacity. The nature of the processes by which $S$ exercises these rules is as yet unclear. Nor is the case for this hypothesis furthered by the fact that, when complexity and length are controlled, passives are recalled as well as, or better than active sentences (Martin and Roberts, 1966).

An alternative proposal is relevant: It has been hypothesized that the speaker is sensitive

1 This research was supported by the Advanced Research Projects Agency, Department of Defense, monitored by Air Force Office of Scientific Research, under Contract Nos. AF 49(638)-1235 and AF 49(638)1736 with the Human Performance Center, Department of Psychology. I thank Edwin Martin for his helpful comments. to informational or contextual constraints on the individual sentence elements (words) in generating a sentence both in speaking and in recall (Deese, 1961; Roberts, 1967). Different syntactic types, e.g., actives and passives, have different amounts and patterns of constraint (Clark, 1965) which lead to the expectation of different patterns of recall errors between the two types. Such differential patterns have been reported for the loss of certain word classes over time (Martin, Roberts and Collins, 1968). The alternative hypothesis, then, is that differential constraints regulate the reconstruction or guessing of stimulus elements in recall.

In order to accentuate the processing effects, constraints that were nonsyntactic in nature were used as a probe. It was hypothesized that associative links between the subject and the object would interact with such syntactic factors as sentence type and complexity. The effectiveness of intra-sentence association strength has been demonstrated (Rosenberg, 1966).

\section{METHOD}

The experiment involved manipulation of the following three factors: Two levels of complexity-mean depths of 1.14 and 1.86 (Martin and Roberts, 1966; Yngve, 1960); two syntactic types-active and passive; and four conditions of association between the logical subject and the logical object (see below). Thirty-two sentences, each seven words long, were constructed, 16 sentences at each level mean depth. Of the 16 , there 
were two of each association condition for each of the two syntactic types. Mean depth was a between-Ss variable; association and sentence type were within-Ss variables.

The association conditions apply to the order of the two nouns within the sentences, these being the logical subject and the logical object. Noun pairs were chosen from the Russell-Jenkins Minnesota norms (1954) to yield the four conditions as follows: $H-\mathrm{N}_{1}$ elicits $\mathrm{N}_{2}$ and $\mathrm{N}_{2}$ elicits $\mathrm{N}_{1}$ (e.g., girl and woman); $L-\mathrm{N}_{1}$ does not elicit $\mathrm{N}_{2}$ and $\mathrm{N}_{2}$ does not elicit $\mathrm{N}_{1}$ (e.g., priest and doctor); $F-\mathrm{N}_{1}$ elicits $\mathrm{N}_{2}$ but $\mathrm{N}_{2}$ does not elicit $\mathrm{N}_{1}$ (e.g., soldier and man); $B-N_{1}$ does not elicit $N_{2}$ but $N_{2}$ elicits $\mathrm{N}_{1}$ (e.g., man and soldier). The only further restriction placed on these pairs was that both $\mathrm{N}_{1}$ and $\mathrm{N}_{2}$ could serve as a logical subject and as a logical object of both an active and a passive sentence, and that none of the nouns chosen were the most frequent associates of the other member of the pair. Thus, the sentence The soldier was watching the old man is designated as an $F$ sentence, mean depth of 1.14, active. Similarly, Men were usually watched by soldiers carefully is a $B$ sentence, mean depth of 1.86 , passive.

Four lists at each depth level were selected, each list containing eight sentences drawn from the 16 . Of the eight sentences, four were active, four passive; and within each syntactic type, each association condition was represented once. The four lists were constructed such that each association combination of $N_{1}$ and $N_{2}$ (of which there were four, $N_{1}$ and $N_{2}$, and $N_{2}$ and $N_{1}$ for both actives and passives) occurred once in each list.

Eighty paid volunteers were randomly assigned to the two mean depth conditions. They were run in groups of five to yield $10 \mathrm{Ss}$ per list. The eight sentences of a given list were read aloud by $E$ in a normal conversational voice; when all sentences had been read, $E$ said "write", and the $S$ s wrote as many of the sentences as they could remember on $3 \times 5$ cards, one sentence per card. The $S$ s were not allowed to look back at what they had alrcady written. This procedure was repcated for eight trials. The order of the association-type combinations was counterbalanced with respect to list position over the eight trials. Two initial orders of each list were used to control partially initial list effects.

\section{RESULTS}

Table 1 shows the proportion of completely correct sentence recalls, collapsed over trials, for the two mean depth levels and the four association conditions. Each proportion is based on $(40 \mathrm{Ss}) \times(8$ trials $)=320$ retention tests. For this measure of performance, there was no difference between the two unidirec- tional conditions and for the purposes of exposition they are collapsed.

TABLE 1

Pfrcent Corrfet Rfcalls in Exp. II (FL)

\begin{tabular}{cccccc}
\hline & \multicolumn{2}{c}{$\mathrm{A}$} & & \multicolumn{2}{c}{$\mathrm{P}$} \\
\cline { 6 - 6 } \cline { 5 - 6 } $\begin{array}{c}\text { Association } \\
\text { Condition }\end{array}$ & 1.14 & 1.86 & & 1.14 & 1.86 \\
\hline $\mathrm{H}$ & .60 & .38 & & .73 & .36 \\
$\mathrm{~L}$ & .58 & .36 & & .68 & .44 \\
$\mathrm{U}^{a}$ & .51 & .29 & & .62 & .30 \\
\hline
\end{tabular}

- Unidirectional associations.

An analysis of variance indicates that mean depth, association condition, syntactic type and trials are all significant factors: $F(1,78)=$ $47.29, \quad F(3,234)=7.94, \quad F(1,78)=8.65$ and $F(7,546)=134.53$, respectively, all with $p<.01$. In addition, only the interactions between trials and mean depth, $F(7,546)=8.14, p<.01$, between type and mean depth, $F(7,78)=4.98$, $p<.05$, were significant.

Analyses within syntactic types, however, are more pertinent to the hypotheses under investigation. For active sentences, mean depth and trials, $F(1,78)=26.71$ and $F(7,546)$ $=71.41$, respectively, both with $p<.01$, were the only significant main effects. Only the interaction between trials and mean depth, $F(7,546)=3.06, p<.01$, was significant. For the passive sentences, mean depth, trials and association, $F(1,78)=53.58, F(7,546)=78.19$, $F(3,234)=5.03$ respectively, all with $p<.01$, were significant factors. Only the interactions between trials and mean depth, $F(7,546)=$ $6.69, p<.01$, and between trials and association, $F(21,1638)=1.83, p<.05$, were significant.

Thus, for completely correct sentence recall, the following variables were effective in the prediction of performance. The higher level of mean depth produces poorer recall and a slower learning rate for both actives and passives than does the lower mean-depth level. Passive sentences are recalled better than active sentences except at the higher meandepth level where actives and passives are 
recalled equally well. The association conditions appear to discriminate among actives and passives in that the unidirectional conditions, relative to the $L$ condition, interfere with recall only in the passives. $\Lambda$ dditionally, trials and association only interact in the passive conditions.

\section{Correct Word Recall}

There were no differences between any of the conditions in the number of nouns, noun pairs, or noun pairs in correct order recalled. In general, nouns and verbs were the parts of speech best recalled, while adverbs were the worst recalled.

\section{Error Analyses}

Out of $(40 \mathrm{Ss}) \times(8$ sentences $) \times(8$ trials $)=$ 5120 recall events, 2708 , or $52.9 \%$, were erroneous in some respect. The proportion of recalls that were "transformations" to other syntactic types were the following: Active-1.14, .020 ; active-1.86, .012; passive-1.14, .018; passive-1.86, .029 . The relative proportion of passives changed to actives is slightly greater than that of actives changed to passives. At least a partial explication of this phenomenon is provided in Table 2, wherein are shown the proportions of all complete-sentence recall errors under a given combination of syntactic type, mean depth, and association condition that were changes in syntactic type. It can be seen that the greater proportion of passiveto-active changes occurred under the unidirec-

TABLE 2

Proportion of Complete Sentfnce Errors Changfid IN TYPE

\begin{tabular}{|c|c|c|c|c|}
\hline \multirow{2}{*}{$\begin{array}{l}\text { Association } \\
\text { Condition }\end{array}$} & \multicolumn{2}{|c|}{ A } & \multicolumn{2}{|c|}{$\mathbf{P}$} \\
\hline & 1.14 & 1.86 & 1.14 & 1.86 \\
\hline F & .21 & .05 & .53 & .33 \\
\hline B & .32 & .10 & .47 & .49 \\
\hline H & .32 & .21 & .34 & .10 \\
\hline $\mathbf{L}$ & .27 & .10 & .33 & .12 \\
\hline Mean & .28 & .11 & .44 & .28 \\
\hline
\end{tabular}

tional association conditions. It can also be seen that fewer changes in syntactic type occur at the higher mean-depth level than at the lower, even though errors in general are more frequent at the higher level.

A particularly instructive pattern of errors is the following. There were certain transformation errors for which the noun order was not changed when the sentence itself was changed from an active to a passive or vice versa. This processing failure reverses the meaning of the sentence. For example, $S$ is presented with Doctors are now interviewing the priests hurriedly, and he responds with Doctorsarenowinterviewed by priests hurriedly. The proportion of all transformation errors that are of this kind at mean depth 1.14 was much lower for passives than for actives, .54 and .86 , respectively. At mean depth 1.86 , the corresponding proportions were less divergent, .69 and .67, respectively. The mean proportion (mean depth 1.14 and 1.86 combined) for the passives was .62 ; for the actives, .80. These results may be summarized by saying that failures to reverse appropriately the two nouns upon changing syntactic type in recall was less prevalent when shifting from the passive to the active type than vice versa, and less prevalent among the higher meandepth sentences. Moreover, while association condition does affect the incidence of shifts in syntactic types at recall (Table 2), association condition does not affect the incidence of failure to reverse the two nouns.

One further type of error analysis was conducted. Even though no difference in proportion of errors of all kinds was found between actives and passives at mean depth 1.86 , the pattern of errors indicates that $S \mathrm{~s}$ responded differentially to these two grammatical forms. Each sentence at mean depth 1.86 contained two adverbs, one in position 3 and one in position 7. There are two possible errors at each position: (a) Omission of the adverb, or (b) substitution of an intrusion for that adverb. Consider only those sentence recalls where only one adverb error was made. 
For the active sentences, $45 \%$ of these adverb errors were omissions of and $35 \%$ were substitutions for the first adverb, compared with 2 and $18 \%$ respectively, for the second adverb. For the passive sentences, first adverb omissions and substitutions were 32 and $61 \%$, respectively, while second adverb omissions and substitutions were 0 and $7 \%$. These percentages are based on approximately 200 observations on each syntactic type. Clearly the first adverb is the more labile. However, the response to the first adverb differs according to the syntactic type of the presented sentence: When a passive sentence was to be recalled, $S$ s tended to substitute for the adverb in position 3; when an active sentence was to be recalled, the tendency was toward omission. Further, the adverb in position 7 is more than twice as labile in the actives than in the passives.

\section{Discussion}

The results indicate that while sentence complexity (as indexed by mean depth) appears to affect sentential retention in general, both syntactic type and subject-object associative relations are significant factors in the recallability of a sentence. Moreover, certain elements (e.g., adverbs) of the to-be-recalled string are more liable to interference-forgetting than are other elements, where lability depends upon grammatical context (syntactic type, mean depth). Associative relations that are unidirectional are likely to interfere with the recall of the complete string, although not with the nouns that stand in that associative relation, if the sentence is passive. This interference does not occur if the sentence is active. However, errors in general are more likely if the sentence is active regardless of associative links between subject and object. ${ }^{2}$

\footnotetext{
2 The same variables, excepting trials, were tested in a Peterson-type short-term memory experiment with the same stimulus materials and a retention interval of $20 \mathrm{sec}$. (Roberts, 1966). All main effects, interactions and lack of interactions, and error analyses mirrored the present findings.
}

It was also observed that $S$ s change actives to passives, and vice versa, but tend not to alter the positions of the logical subject and logical object in such a way as to maintain semantic equivalence. A ramification of this high incidence of nonreversal errors within transformational errors is worth pointing out. Most studies in the recall of sentences have not used reversible logical subjects and logical objects. The fact that these studies (e.g., Mehler, 1963; Martin and Roberts, 1966) have not obtained changes in sentence kind without positional reversal of the subject and object would imply that $S$ s utilize some sort of semantic scanning and correction procedure before the actual response is emitted, thus implicating an edited construction process of some sort (Deese, 1961 ; Peterson, 1967).

\section{REFERENCES}

Clark, H. Some structural properties of simple active and passive sentences. J. verb. Learn. verb. Behav., $1965,4,365-370$.

DEESE, J. From the isolated verbal unit to connected discourse. In C. N. Cofer (Ed.), Verbal Learning and Verbal Behavior. New York: McGraw-Hill, 1961, Pp. 11-30.

MARTIN, E. AND RoberTs, K. H. Grammatical factors in sentence retention. J. verb. Learn. verb. Behav., 1966, 5, 211-218.

Martin, E., Roberts, K. H., AND Collins, A. Shortterm memory for sentences. J. verb. Learn. verb. Behav., 1968, 7, 560-566.

MEHLER, J. Some effects of grammatical transformations on the recall of English sentences. J. verb. Learn. verb. Behav., 1963, 2, 346-351.

Peterson, L. R. Search and judgement in memory. In B. Kleinmuntz (Ed.), Concepts and the Structure of Memory. New York: Wiley, 1967.

ROBERTS, K. H. The interaction of normative associations and grammatical factors sentence in retention. Paper read at meeting of Mid-western Psychological Association, May, 1966.

RoBERTS, K. H. Some effects of grammatical context on the recall of sentence elements. Paper read at meeting of Mid-western Psychological Association, May, 1967.

ROSENBERG, S. Recall of sentences as a function of syntactic and associative habits. $J$. verb. Learn. verb. Behav., 196 €.5 392-396. 
Russell, W. A. and Jenkins, J. J. The complete Minnesota norms for responses to 100 words from the Kent-Rosonofr Word Association Test. Tech. Rep. No. 11, 1954, Contract No. N8onr66216, Office of Naval Research and Univer. of Minnesota.
YNGVE, V. H. A model and an hypothesis for language structure. Proc. Amer. Phil. Soc., 1960, 104, 444-466.

(Received March 8, 1968) 